\title{
Failure to Shock with Inadequate Defibrillator Threshold Safety Margin
}

National Cancer Institute

\section{Source}

National Cancer Institute. Failure to Shock with Inadequate Defibrillator Threshold Safety

Margin. NCl Thesaurus. Code C99954.

The cardiac lead failed to administer a shock and had an inadequate defibrillator threshold safety margin. (ACC) 